\title{
SHARP-INTERFACE PHASE-CHANGE MODEL WITH THE VOF METHOD
}

\author{
Lubomír Bureš $^{1, *, 2, *}$ \& Yohei Sato ${ }^{1}$ \\ ${ }^{1}$ Nuclear Energy and Safety, Paul Scherrer Institute, Villigen, 5232, Switzerland \\ ${ }^{2}$ Swiss Federal Institute of Technology, Lausanne, 1015, Switzerland
}

\begin{abstract}
Proper resolution of phasic interfaces is a key requirement for performing direct numerical simulations (DNS) of multiphase flows. The Volume-of-Fluid method (VOF) represents the phases in terms of a cell-wise volume-fraction function. VOF can capture the interface in a sharp manner with subgrid accuracy, while being inherently mass conservative at the same time. For multiphase problems involving phase change, the temperature field near the interface must also be resolved in a sharp way, accounting for interfacial jump conditions. Using an irregular stencil dependent on the interface position, the temperature gradients at the interface can be captured. In turn, they can be used for calculating interfacial mass transfer rate. In this work, a coupling of a sharp-interface phase-change model and the VOF method is developed. Both the interfacial position and the temperature field are resolved with subgrid accuracy. Mass source due to phase change is localised exactly at the interface and possible variability of the interfacial temperature is accounted for. Coupling with conjugate heat transfer is implemented and subgrid interfacial profiles in the vicinity of the solid are explicitly captured. The methodology implementation is verified and validated using standard benchmarks. Simulations of rising bubbles and nucleate boiling are used for validation for problems involving phase transition. To the best of authors' knowledge, there has not yet been an attempt to validate a sharp phase-change model with a geometric VOF interface tracking method. Therefore, this work represents another step towards high-fidelity DNS of flows with phase transition.
\end{abstract}

KEY WORDS: phase change, boiling flows, VOF, direct numerical simulation, interface tracking

\section{INTRODUCTION}

Diabatic flows involving phase change are a special case of multiphase flows, which finds use in a very wide palette of applications. Due to their ubiquitous nature and the role they play in industrial systems, attaining the understanding of mechanisms involved in these flows is of high importance, both from a fundamental and applied perspective. Traditionally, experimental work has been the main tool for investigating fluid flows; at the same time, the complexity of multiphase diabatic flows limits the amount of information that can be extracted from an experiment even with the most advanced experimental setup.

Numerical simulations provide a direct way of analysing complex physical phenomena with the possibility to quantify the significance of their individual aspects. Direct Numerical Simulation (DNS) attempts to resolve the equations of fluid continuum mechanics as they are without the need for turbulence modelling; therefore, use of DNS brings insight into the mechanisms behind multiphase flow problems involving phase change. Together with experimental observations, DNS aids in understanding these mechanisms on a fundamental level. 
In order to perform DNS of multiphase flows, the liquid-vapour interface must be captured using an Interface Tracking Method (ITM). A prominent example of an ITM is the Volume-of-Fluid (VOF) method [4], in which the phases are represented in terms of a cell-wise phasic volume-fraction function. VOF with geometric advection techniques can capture the interface in a sharp manner with subgrid accuracy, while being inherently mass conservative at the same time. In the recent years, significant improvement has been achieved in development of techniques for capturing interfacial characteristics using VOF, especially the surface tension force [9].

For preserving subgrid accuracy in problems involving phase change, a sharp-interface phase-change model must also be considered. In this model, the temperature field near the interface is resolved in a sharp way, accounting for interfacial jump conditions. Using an irregular stencil dependent on the interface position, the temperature gradients at the interface can be captured. In turn, they can be used for calculating interfacial mass transfer rate. Using this model, mass source due to phase change can be localised exactly at the interface and is included in the VOF algorithm.

Geometric VOF with subgrid-accurate phase change has received some attention in the past: 2D simulations of film boiling were conducted by the group of Welch $[1,15,16]$; in these works, the irregular stencil for temperature gradients was used only during the mass transfer rate calculations. In the work by Schlottke and Weigand [12] and subsequent works related to the DNS code FS3D [2], the mass transfer rate is calculated from the species concentration gradient. In [7], the implementation of geometric VOF with subgrid-accurate phase change was verified using a 1D Stefan problem and simulation of 3D bubble growth in quiescent liquid.

In this work, a coupling of the geometric VOF method of Kawano [5] with the sharp-interface phase-change model of Sato and Niceno [11] was developed and implemented in the framework of the PSI-BOIL CFD code [8]. The developed method was subjected to the standard procedure of computer code verification and validation to establish its credibility; for verification purposes, several problems including the Stefan problem and the Sucking problem were considered. For validation purposes, bubble growth in the presence of gravity was simulated, as well as nucleate boiling under high pressures. For the purposes of the latter, coupling with conjugate heat transfer was implemented and interfacial profiles and temperature field in the vicinity of the solid are explicitly captured with subgrid accuracy.

\section{RESULTS}

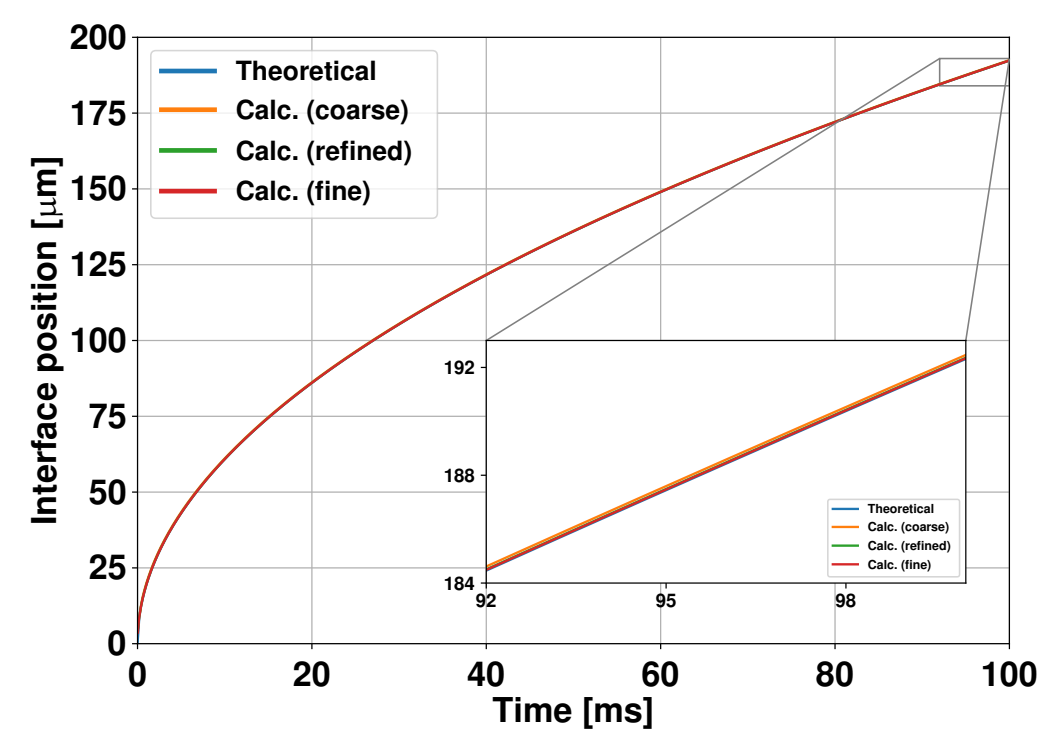

Fig. 1 Interface position as a function time for the Stefan problem. 
TFEC-2020-31939

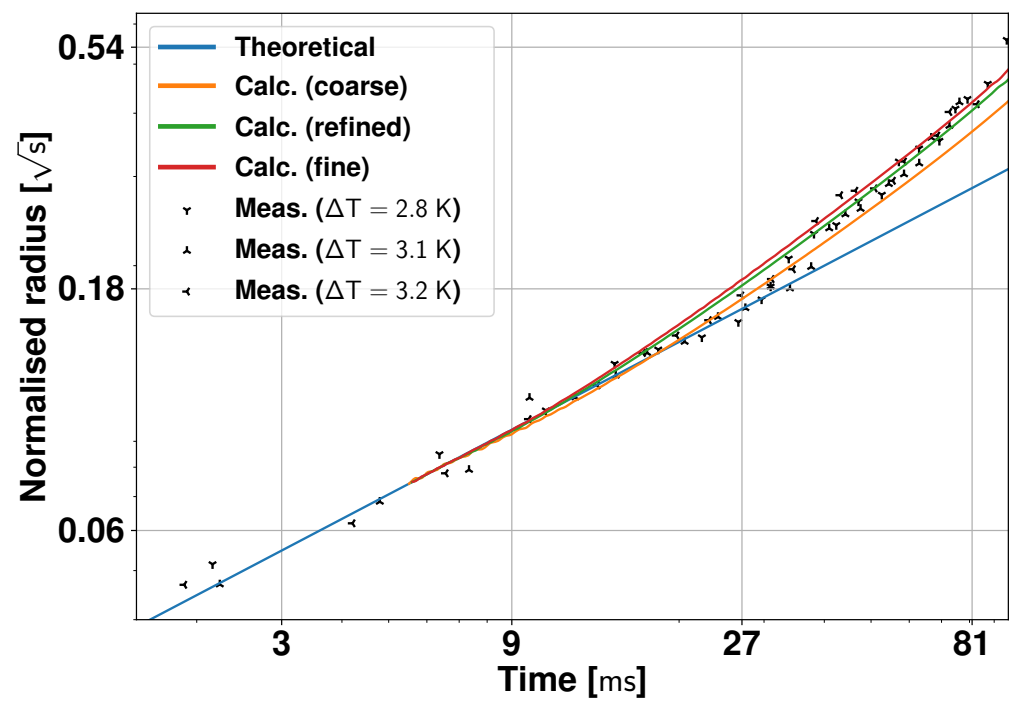

Fig. 2 Normalised bubble radius from measurements [3], theoretical predictions [13], and PSI-BOIL calculations as functions of time. For the latter, $\Delta T=3.1 \mathrm{~K}$.

For verification purposes, the Stefan problem [14] and the Sucking problem [16] were simulated with the same initial and boundary conditions as used in [11]. For the Stefan problem, the agreement of the calculated interfacial position with the theoretical prediction was near-perfect; the grid convergence rate was calculated using the time-integrated L2-norm error of the interfacial position as $\sim \Delta x^{1.69}$. For the Sucking problem, the temperature distribution at $t=0.5 \mathrm{~s}$ was used to compare the results against theory. Very good agreement was achieved and the space-integrated L2-norm error of the temperature distribution scaled with grid spacing as $\sim \Delta x^{1.25}$. Fig. 1 presents the interface position as a function time for the Stefan problem for three levels of grid refinement.

For validation purposes, a three-dimensional simulation of a rising vapour bubble in superheated liquid under gravity was carried out with the same initial and boundary conditions as used in [11]. Fig. 2 presents the comparison of calculated normalised bubble radius at three levels of grid refinement with the zero-gravity

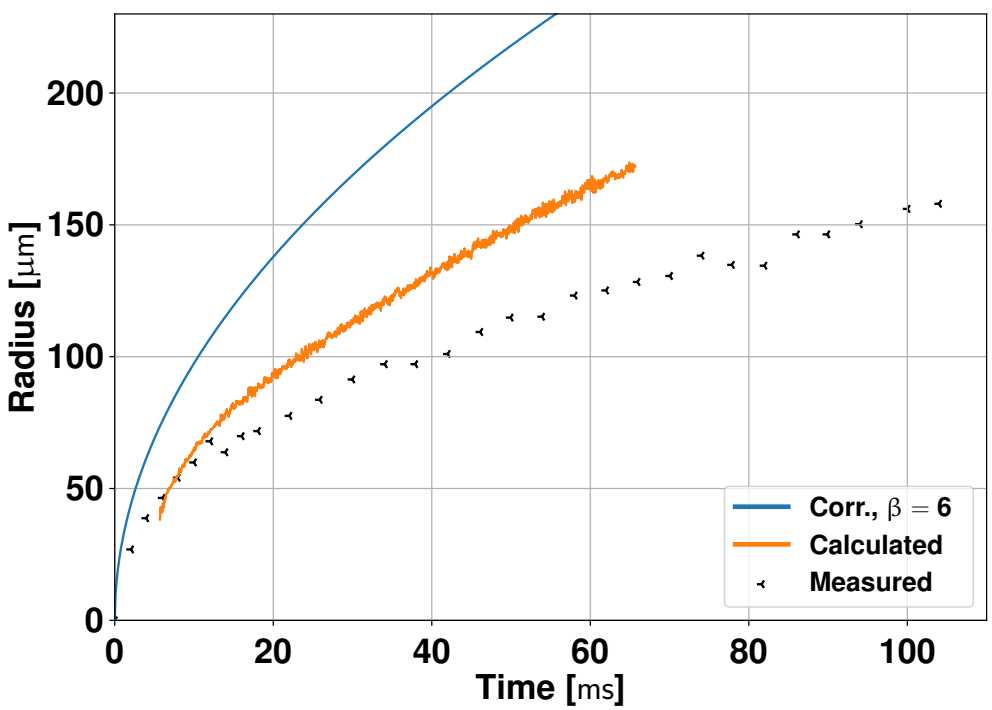

Fig. 3 Primary bubble radius from measurements [10], empirical correlation predictions [6], and PSI-BOIL calculations as functions of time. 
solution of Scriven [13], measurement [3]. It can be observed that reasonable agreement with experimental data has been achieved with satisfactory grid convergence.

Subsequently, saturated pool boiling of water at 22.3 bar was simulated. The comparison of the calculated evolution of the primary bubble radius with experimental data of Sakashita [10] and the prediction from the correlation of Labuntsov [6] is shown in Fig. 3. It can be seen that the lift-off diameter is calculated accurately, while the bubble growth rate is overestimated; the reason for the discrepancy is currently under investigation.

Overall, the considered benchmarks verify the developed method and indicate its capacity to simulate nontrivial problems with respectable accuracy. The work performed thus represents the first example of validation of a geometric VOF method with subgrid-accurate phase change implemented into a DNS code. Therefore, this work represents another step towards high-fidelity DNS of flows with phase transition.

\section{ACKNOWLEDGMENTS}

This work is supported by the Swiss National Science Foundation (SNSF) under Grant No. 200021_175893.

\section{REFERENCES}

[1] Agarwal, D. K., Welch, S. W. J., Biswas, G., and Durst, F., "Planar simulation of bubble growth in film boiling in near-critical water using a variant of the vof method," Journal of Heat Transfer, 126(3), pp. 329-338, (2004).

[2] Eisenschmidt, K., Ertl, M., Gomaa, H., Kieffer-Roth, C., Meister, C., Rauschenberger, P., Reitzle, M., Schlottke, K., and Weigand, B., "Direct numerical simulations for multiphase flows: An overview of the multiphase code fs3d," Applied Mathematics and Computation, 272, pp. 508-517, (2016).

[3] Florschuetz, L., Henry, C., and Khan, A., "Growth rates of free vapor bubbles in liquids at uniform superheats under normal and zero gravity conditions," International Journal of Heat and Mass Transfer, 12(11), pp. 1465 - 1489, (1969).

[4] Hirt, C. and Nichols, B., "Volume of fluid (vof) method for the dynamics of free boundaries," Journal of Computational Physics, 39(1), pp. 201-225, (1981).

[5] Kawano, A., "A simple volume-of-fluid reconstruction method for three-dimensional two-phase flows," Computers \& Fluids, 134-135, pp. 130-145, (2016).

[6] Labuntsov, D., "Study of the growth of bubbles during boiling of saturated water within a wide range of pressures by means of high-speed moving pictures," Teplofizika Vysokikh Temperatur, 2(3), pp. 446-453, (1964).

[7] Malan, L., Direct numerical simulation of free-surface and interfacial flow using the VOF method : cavitating bubble clouds and phase change, Thesis, Université Pierre et Marie Curie - Paris VI, (2017).

[8] Niceno, B., Sato, Y., Badillo, A., and Andreani, M., "Multi-scale modeling and analysis of convective boiling: Towards the prediction of chf in rod bundles," Nuclear Engineering and Technology, 42(6), pp. 620-635, (2010).

[9] Popinet, S., "Numerical models of surface tension," Annual Review of Fluid Mechanics, 50(1), pp. 49-75, (2018).

[10] Sakashita, H., "Bubble growth rates and nucleation site densities in saturated pool boiling of water at high pressures," Journal of Nuclear Science and Technology, 48(5), pp. 734-743, (2011).

[11] Sato, Y. and Niceno, B., "A sharp-interface phase change model for a mass-conservative interface tracking method," Journal of Computational Physics, 249, pp. 127-161, (2013).

[12] Schlottke, J. and Weigand, B., "Direct numerical simulation of evaporating droplets," Journal of Computational Physics, 227(10), pp. 5215-5237, (2008).

[13] Scriven, L., "On the dynamics of phase growth," Chemical Engineering Science, 10(1), pp. 1 - 13, (1959).

[14] Son, G. and Dhir, V. K., "Numerical Simulation of Film Boiling Near Critical Pressures With a Level Set Method," Journal of Heat Transfer, 120(1), pp. 183-192, (1998).

[15] Welch, S. W. J. and Rachidi, T., "Numerical computation of film boiling including conjugate heat transfer," Numerical Heat Transfer, Part B: Fundamentals, 42(1), pp. 35-53, (2002).

[16] Welch, S. W. J. and Wilson, J., "A volume of fluid based method for fluid flows with phase change," Journal of Computational Physics, 160(2), pp. 662-682, (2000). 